\title{
A Mechanical Quadrature Method for Solving Delay Volterra Integral Equation with Weakly Singular Kernels
}

\author{
Li Zhang, Jin Huang (D), Yubin Pan (D), and Xiaoxia Wen \\ School of Mathematical Sciences, University of Electronic Science and Technology of China, Chengdu, Sichuan 611731, China \\ Correspondence should be addressed to Jin Huang; huangjin12345@163.com
}

Received 15 February 2019; Accepted 21 May 2019; Published 16 June 2019

Academic Editor: Danilo Comminiello

Copyright (C) 2019 Li Zhang et al. This is an open access article distributed under the Creative Commons Attribution License, which permits unrestricted use, distribution, and reproduction in any medium, provided the original work is properly cited.

\begin{abstract}
In this work, a mechanical quadrature method based on modified trapezoid formula is used for solving weakly singular Volterra integral equation with proportional delays. An improved Gronwall inequality is testified and adopted to prove the existence and uniqueness of the solution of the original equation. Then, we study the convergence and the error estimation of the mechanical quadrature method. Moreover, Richardson extrapolation based on the asymptotic expansion of error not only possesses a high accuracy but also has the posterior error estimate which can be used to design self-adaptive algorithm. Numerical experiments demonstrate the efficiency and applicability of the proposed method.
\end{abstract}

\section{Introduction}

In recent years, Volterra integral equation with delay has received a considerable amount of attention. This paper considers the following weakly singular Volterra integral equations with proportional delays:

$$
u(t)=g(t)+(I u)(t)+\left(I_{\theta} u\right)(t), \quad t \in I=[0, T],
$$

with

$$
\begin{gathered}
(I u)(t):=\int_{0}^{t} K_{1}(t, s) u(s) d s, \\
\left(I_{\theta} u\right)(t):=\int_{0}^{\theta(t)} K_{2}(t, s) u(s) d s,
\end{gathered}
$$

and

$$
\begin{aligned}
\theta(t) & :=q t, \quad 0<q<1, \\
K_{1}(t, s) & :=s^{\alpha} k_{1}(t, s), \\
K_{2}(t, s) & :=s^{\gamma} k_{2}(t, s), \\
& \quad-1<\alpha<0,-1<\gamma \leq 0,
\end{aligned}
$$

where $k_{1}(t, s)$ and $k_{2}(t, s)$ are known continuous functions defined on the domains $D:=\{(t, s): 0 \leq s \leq t \leq T\}$ and
$D_{\theta}:=\{(t, s): 0 \leq s \leq \theta(t), t \in I=[0, T]\}$, respectively. $g(t)$ is a known function and $u(t)$ is an unknown function. In practice, the delay arguments are consistent with the real phenomena which make the models more realistic for simulation. Delay integral equation and partial differential equation have been widely used in many population growth and relevant phenomena in mathematical biology [1-3].

Numerical algorithms for implementing delay models should be designed specially according to the nature of the equations. There are many numerical techniques for the delay differential equations [4-8] and integral-differential equations [9-11]. Simultaneously, vast researchers also focused their interests on the numerical techniques of delay integral equations with continuous kernels, such as least squares approximation method [12], spectral method [13], Bernoulli wavelet method [14], and collocation method [15]. Xie et al. in [16] handled the Volterra integral equation with the delay function vanishing at the initial point in the given interval; they found that the iterated collocation solution possessed local superconvergence at the mesh points. In [17], the authors adopted multistep method based on Hermite collocation method and it turned out that the numerical method had uniform order $2 m+2 r$ with $m$ collocation points and $r$ previous time steps. In [18], an hp-spectral collocation method was used for nonlinear Volterra integral equations with vanishing variable delays. 
There are a few researches on the delay integral equation with weakly singular kernels, such as [19]. In this paper, we concentrate on (1) whose upper limit of integral is a delay function and the integral kernels are weakly singular functions, which increase the computational complexity and the theoretical difficulty. To the best of our knowledge, there are no studies in (1) by the mechanical quadrature method in recent years. A further advantage of this method for (1) is that the error has an asymptotic expansion. Thus, we can improve the accuracy order of approximation. Simultaneously, the theoretical analysis is complete and the calculation is simplified.

In this paper, we firstly deduce the improved Gronwall inequality; the existence and uniqueness of the solution for (1) are testified via the improved Gronwall inequality. Then the equation is approximated by employing the floor technique to the delay argument $\theta(t)$ and by adopting the quadrature formula [20] to the weakly integrals. Next, the approximate equation for (1) is constructed by combining the mechanical quadrature method and the interpolation technique; then, we use the iterative method for solving the approximate equation. The existence and uniqueness of the solution for the approximate equation are testified via the discrete Gronwall inequality. Finally, we prove that the convergent order is $O\left(h^{2+\min (\alpha, \gamma)}\right)$. In order to achieve a higher accuracy order $O\left(h^{2}\right)$, the Richardson- $h^{2+\min (\alpha, \gamma)}$ extrapolation based on the asymptotic expansion of error is adopted; moreover, a posterior error estimate is realized conveniently.

The layouts of this paper are as follows. In Section 2, we prove the existence and uniqueness of the solution for (1). In Section 3, we introduce the quadrature method, iterative method, and interpolation technique. In Section 4, the existence and uniqueness of the solution for the approximate equation are discussed. In Section 5, the convergence and the error estimation are obtained to ensure the reliability of the method. In Section 6, the asymptotic expansion of the error is achieved, a higher accuracy order is realized by extrapolation, and a posterior error estimate is derived. In Section 7, some numerical examples are demonstrated to illustrate the theoretical results. Some concluding remarks are provided in Section 8.

\section{The Existence and Uniqueness of the Solution for the Original Equation}

In this section, we will verify the existence and uniqueness of the solution for (1). We first prove the improved Gronwall inequality.

Lemma 1. Suppose that $g(t), h(t)$, and $u(t)$ are nonnegative integrable functions, $t \in[0, T], 0<q<1$, and $A \geq 0$; based on the inequality

$$
u(t) \leq A+\int_{0}^{t} g(s) u(s) d s+\int_{0}^{q t} h(s) u(s) d s,
$$

we have

$$
u(t) \leq A e^{\int_{0}^{t}(g(s)+h(s)) d s}
$$

Proof. Due to the fact that $0<q<1$, qt $<t$ and

$$
\begin{aligned}
u(t) & \leq A+\int_{0}^{t} g(s) u(s) d s+\int_{0}^{t} h(s) u(s) d s \\
& \leq A+\int_{0}^{t}(g(s)+h(s)) u(s) d s .
\end{aligned}
$$

Let $H(s)=g(s)+h(s)$; we can deduce

$$
\frac{H(t) u(t)}{A+\int_{0}^{t} H(s) u(s) d s} \leq H(t) .
$$

Integrate on both sides

$$
\ln \left(A+\int_{0}^{t} H(s) u(s) d s\right)-\ln A \leq \int_{0}^{t} H(s) d s
$$

and then

$$
A+\int_{0}^{t} H(s) u(s) d s \leq A e^{\int_{0}^{t} H(s) d s}
$$

namely,

$$
u(t) \leq A e^{\int_{0}^{t} H(s) d s} .
$$

The proof of the Lemma 1 is completed.

Theorem 2. Assume that $k_{i}(t, s)(i=1,2)$ are known continuous functions defined on the domains $D:=\{(t, s): 0 \leq$ $s \leq t \leq T\}$ and $D_{\theta}:=\{(t, s): 0 \leq s \leq \theta(t), t \in I=[0, T]\}$, respectively; then the solution of (1) is existent uniquely.

Proof. We construct the sequence $u_{k}(t)(k \in \mathbf{N})$, satisfying

$$
\begin{aligned}
u_{0}(t)= & u(0)=g(t), \\
u_{k}(t)= & g(t)+\int_{0}^{t} K_{1}(t, s) u_{k-1}(s) d s \\
& +\int_{0}^{q t} K_{2}(t, s) u_{k-1}(s) d s,
\end{aligned}
$$

where $K_{1}(t, s)$ and $K_{2}(t, s)$ are defined in (3), with $k \in \mathbf{N}$. $k_{i}(t, s)(i=1,2)$ are continuous functions; then there exists a constant $C$ such that $\left|k_{i}(t, s)\right| \leq C$. We have

$$
\begin{aligned}
& \left|u_{2}(t)-u_{1}(t)\right| \\
& \leq C \int_{0}^{t} s^{\alpha}\left|u_{1}(s)-u_{0}(s)\right| d s \\
& \quad+C \int_{0}^{q t} s^{\gamma}\left|u_{1}(s)-u_{0}(s)\right| d s \\
& \leq C \int_{0}^{t}\left[s^{\alpha}\left|u_{1}(s)-u_{0}(s)\right|+s^{\gamma}\left|u_{1}(s)-u_{0}(s)\right|\right] d s \\
& \leq C a \int_{0}^{t}\left(s^{\alpha}+s^{\gamma}\right) d s \leq 2 C a \int_{0}^{t} s^{\mu} d s=2 C a \frac{t^{\mu+1}}{\mu+1},
\end{aligned}
$$


with $a=\max _{0 \leq t \leq T}\left|u_{1}(t)-u_{0}(t)\right| ; \mu=\min \{\alpha, \gamma\}$. Now, we can deduce

$$
\left|u_{k}(t)-u_{k-1}(t)\right| \leq a \frac{(2 C)^{k-1}}{(k-1) !(\mu+1)^{k-1}} t^{(k-1)(\mu+1)} .
$$

By means of the mathematical induction, when $n=k+1$, we obtain

$$
\begin{aligned}
& \left|u_{k+1}(t)-u_{k}(t)\right| \leq C \int_{0}^{t} s^{\alpha}\left|u_{k}(s)-u_{k-1}(s)\right| d s \\
& +C \int_{0}^{q t} s^{\gamma}\left|u_{k}(s)-u_{k-1}(s)\right| d s \\
& \quad \leq C \int_{0}^{t}\left[s^{\alpha}\left|u_{k}(s)-u_{k-1}(s)\right|\right. \\
& \left.\quad+s^{\gamma}\left|u_{k}(s)-u_{k-1}(s)\right|\right] d s \leq 2 C \int_{0}^{t} s^{\mu} \mid u_{k}(s) \\
& \quad-u_{k-1}(s) \mid d s \leq a \frac{(2 C)^{k}}{k !(\mu+1)^{k}} t^{k(\mu+1)} .
\end{aligned}
$$
in fact,

Next, we prove that $u_{k}(t)$ is the basic sequence in $C[0, T]$;

$$
\begin{aligned}
& \left|u_{k}(t)-u_{k+m}(t)\right| \\
& \leq\left|u_{k+1}(t)-u_{k}(t)\right|+\left|u_{k+2}(t)-u_{k+1}(t)\right|+\cdots \\
& \quad+\left|u_{k+m}(t)-u_{k+m-1}(t)\right| \\
& \leq a \frac{(2 C)^{k}}{k !(\mu+1)^{k}} t^{k(\mu+1)}+\cdots \\
& \quad+a \frac{(2 C)^{k+m-1}}{(k+m-1) !(\mu+1)^{k+m-1}} t^{(k+m-1)(\mu+1)} .
\end{aligned}
$$

For sufficiently small $\epsilon>0$, there exists a positive integer $N$ such that when $n>N$ and any $m>0$, we have

$$
\left|u_{n}(t)-u_{n+m}(t)\right| \leq \epsilon .
$$

According to Cauchy's test for convergence, the sequence $u_{n}(t)(n \in \mathbf{N})$ is convergent uniformly to $u(t)$ which is the solution of (1).

Suppose that both $u$ and $v$ are the solutions of (1); let $|w|=$ $|u-v|$; then we get

$$
\begin{aligned}
|w(t)| & \\
= & \left|\int_{0}^{t} s^{\alpha} k_{1}(t, s) w(s) d s+\int_{0}^{q t} s^{\gamma} k_{2}(t, s) w(s) d s\right| \\
\leq & \int_{0}^{t} s^{\alpha}\left|k_{1}(t, s)\right||w(s)| d s \\
& \quad+\int_{0}^{q t} s^{\gamma}\left|k_{2}(t, s)\right||w(s)| d s
\end{aligned}
$$

$$
\begin{aligned}
& \leq \int_{0}^{t}\left(s^{\alpha}\left|k_{1}(t, s)\right||w(s)|+s^{\gamma}\left|k_{2}(t, s)\right|\right)|w(s)| d s \\
& \leq \int_{0}^{t}\left(s^{\alpha}\left|k_{1}(t, s)\right|+s^{\gamma}\left|k_{2}(t, s)\right|\right)|w(s)| d s .
\end{aligned}
$$

Next, we verify that $s^{\alpha}\left|k_{1}(t, s)\right|+s^{\gamma}\left|k_{2}(t, s)\right|$ is integrable;

$$
\begin{aligned}
& \int_{0}^{t} s^{\alpha}\left|k_{1}(t, s)\right|+s^{\gamma}\left|k_{2}(t, s)\right| d s \leq C \int_{0}^{t}\left(s^{\alpha}+s^{\gamma}\right) d s \\
& \quad \leq C\left(\frac{t^{1+\alpha}}{1+\alpha}+\frac{t^{1+\gamma}}{1+\gamma}\right) .
\end{aligned}
$$

According to Lemma 1, we can derive that $|w(t)|=0$; the solution is unique.

\section{The Quadrature Method and the Iterative Algorithm}

Let the delay function $\theta(t)=t-\tau(t) \geq 0, t \in[0, T]$, satisfy the following conditions [21]:

(1) $\tau(0)=0$, and $\tau(t)>0$ with $t \in(0, T]$ (vanishing delay);

(2) $\theta(t) \leq q_{1} t$ on $I=[0, T]$ for some $q_{1} \in(0,1)$ and $\theta^{\prime}(t) \geq q_{0}>0 ; q_{i}(i=0,1)$ are constants;

(3) $\theta(t) \in C^{1}(I)$.

For $0<q<1$, the special case is $\tau(t)=(1-q) t$; we get $\theta(t)=t-\tau(t)=q t$; (1) is a weakly integral equation with vanishing delay. In order to solve (1), the modified trapezoid quadrature formula is considered to deal with the integrals $(I u)(t)$ and $\left(I_{\theta} u\right)(t)$.

It is challenging because the integral upper limit is a delay function $\theta(t)$ and the integral kernels are weakly singular at the endpoint.

Lemma 3 (see [22]). Let $I(G)=\int_{a}^{b} G(x) d x=\int_{a}^{b}(x-$ $a)^{\alpha} g(x) d x,-1<\alpha<0$, and $g(x) \in C^{2 m}[a, b]$; then the modified trapezoidal rule is

$$
Q_{N}(G)=-h^{(1+\alpha)} g(a) \zeta(-\alpha)+h \sum_{i=1}^{N-1} G\left(x_{i}\right)+\frac{h}{2} G(b),
$$

where $\zeta$ is the zeta function. Further, $I(G)$ has the following asymptotic expansion:

$$
\begin{aligned}
E_{N}(G)= & Q_{N}(G)-I(G) \\
= & \sum_{j=1}^{m-1} \frac{B_{(2 j)}}{(2 j) !} G^{(2 j-1)}(b) h^{2 j} \\
& +\sum_{j=1}^{2 m-1} \frac{(-1)^{j} \zeta(-\alpha-j) g^{(j)}(a) h^{j+\alpha+1}}{j !} \\
& +O\left(h^{2 m}\right),
\end{aligned}
$$

where $B_{(2 j)}, j=1, \ldots, m-1$, are the Bernoulli numbers. 
Lemma 4. Let $u \in C^{3}[0,1]$, and $z=\beta x+(1-\beta) y$ with $\beta \in[0,1], x, y \in[0, T]$; then there is

$$
\begin{aligned}
u(z)= & \beta u(x)+(1-\beta) u(y) \\
& -\frac{\beta(1-\beta)}{2}(x-y)^{2} u^{\prime \prime}(z)+O\left((x-y)^{3}\right) .
\end{aligned}
$$

Proof. The Taylor expansion of function $u(x)$ at the point $z$ is

$$
\begin{aligned}
u(x)= & u(\beta x+(1-\beta) x) \\
= & u(\beta x+(1-\beta) y+(1-\beta)(x-y)) \\
= & u(z+(1-\beta)(x-y)) \\
= & u(z)+(1-\beta)(x-y) u^{\prime}(z) \\
& +\frac{(1-\beta)^{2}}{2}(x-y)^{2} u^{\prime \prime}(z)+O\left((x-y)^{3}\right) .
\end{aligned}
$$

Similarly, the Taylor expansion of function $u(y)$ at the point $z$ is

$$
\begin{aligned}
u(y)= & u(z-\beta(x-y)) \\
= & u(z)-\beta(x-y) u^{\prime}(z)+\frac{\beta^{2}}{2}(x-y)^{2} u^{\prime \prime}(z) \\
& +O\left((x-y)^{3}\right) .
\end{aligned}
$$

Combining (22) with (23), we derive (21).

Now, the concrete approximate scheme of (1) will be constructed on the basis of the above lemmas. We divide $I \in[0, T]$ into $N$ subintervals with the uniform step size $h=T / N ; t_{i}=i h, i=0, \ldots, N$. Let $t$ be replaced by $t_{i}$ in (1); that is to say,

$$
\begin{aligned}
u\left(t_{i}\right)= & g\left(t_{i}\right)+\int_{0}^{t_{i}} s^{\alpha} k_{1}\left(t_{i}, s\right) u(s) d s \\
& +\int_{0}^{q t_{i}} s^{\gamma} k_{2}\left(t_{i}, s\right) u(s) d s \\
= & g\left(t_{i}\right)+\int_{0}^{t_{i}} s^{\alpha} k_{1}\left(t_{i}, s\right) u(s) d s \\
& +\int_{0}^{t_{[q]}} s^{\gamma} k_{2}\left(t_{i}, s\right) u(s) d s \\
& +\int_{t_{[q i]}^{q}}^{q t_{i}} s^{\gamma} k_{2}\left(t_{i}, s\right) u(s) d s \\
= & g\left(t_{i}\right)+I_{1}+I_{2}+I_{3},
\end{aligned}
$$

where $[q i]$ denotes the maximum integer less than $q i$. It is obvious that $u\left(q t_{i}\right), 0<q<1$, are not the node values, and we can turn $u\left(q t_{i}\right)$ to the adjacent points $u\left(t_{[q i]}\right)$ and $u\left(t_{[q i]+1}\right)$ by the linear interpolation approximation with $t_{[q i]} \leq q t_{i} \leq$ $t_{[q i]+1}$. Then, we have that $q t_{i}=\beta_{i} t_{[q i]}+\left(1-\beta_{i}\right) t_{[q]+1}$ are established for $\beta_{i} \in[0,1]$ with $\beta_{i}=1+[q i]-q i$, and

$$
u\left(q t_{i}\right)=\beta_{i} u\left(t_{[q i]}\right)+\left(1-\beta_{i}\right) u\left(t_{[q i]+1}\right) .
$$

Now, we structure the quadrature algorithm; by Lemma 3 and the trapezoidal quadrature rule, we obtain

$$
\begin{aligned}
I_{1} & =\int_{0}^{t_{i}} s^{\alpha} k_{1}\left(t_{i}, s\right) u(s) d s=h \sum_{k=1}^{i-1} t_{k}^{\alpha} k_{1}\left(t_{i}, t_{k}\right) u\left(t_{k}\right) \\
& +\frac{h}{2} t_{i}^{\alpha} k_{1}\left(t_{i}, t_{i}\right) u\left(t_{i}\right)-\zeta(-\alpha) h^{1+\alpha} k_{1}\left(t_{i}, t_{0}\right) u\left(t_{0}\right) \\
& +E_{1, i}\left(s^{\alpha} k_{1}(t, s)\right), \\
I_{2}= & \int_{0}^{t_{[q i]}} s^{\gamma} k_{2}\left(t_{i}, s\right) u(s) d s=h \sum_{k=1}^{[q i]-1} t_{k}^{\gamma} k_{2}\left(t_{i}, t_{k}\right) \\
& \cdot u\left(t_{k}\right)+\frac{h}{2} t_{[q i]}^{\gamma} k_{2}\left(t_{i}, t_{[q i]}\right) u\left(t_{[q i]}\right)-\zeta(-\gamma) \\
& \cdot h^{1+\gamma} k_{2}\left(t_{i}, t_{0}\right) u\left(t_{0}\right)+E_{2, i}\left(s^{\gamma} k_{2}(t, s)\right), \\
I_{3}= & \int_{t_{[q i]}}^{q t_{i}} s^{\gamma} k_{2}\left(t_{i}, s\right) u(s) d s \\
& =\frac{q t_{i}-t_{[q i]}}{2}\left[t_{[q i]}^{\gamma} k_{2}\left(t_{i}, t_{[q i]}\right) u\left(t_{[q i]}\right)+\left(q t_{i}\right)^{\gamma}\right. \\
& \left.\cdot k_{2}\left(t_{i}, q t_{i}\right) u\left(q t_{i}\right)\right]+E_{3, i}\left(s^{\gamma} k_{2}(t, s)\right. \\
& =\frac{q t_{i}-t_{[q i]}}{2}\left[t_{[q i]}^{\gamma} k_{2}\left(t_{i}, t_{[q i]}\right) u\left(t_{[q i]}\right)+\left(q t_{i}\right)^{\gamma}\right. \\
& \left.\cdot k_{2}\left(t_{i}, q t_{i}\right)\left(\beta_{i} u\left(t_{[q i]}\right)+\left(1-\beta_{i}\right) u\left(t_{[q i]+1}\right)\right)\right] \\
& +E_{3, i}\left(s^{\gamma} k_{2}(t, s)\right),
\end{aligned}
$$

where $E_{1, i}, E_{2, i}$, and $E_{3, i}$ are error functions, which are, respectively, equal to

$$
\begin{aligned}
E_{1, i} & =\left.\left[k_{1}\left(t_{i}, s\right) u(s)\right]^{\prime}\right|_{s=0} \zeta(-\alpha-1) h^{2+\alpha} \\
& +\frac{\left.\left[k_{1}\left(t_{i}, s\right) u(s)\right]^{\prime}\right|_{s=0}}{2 !} \zeta(-\alpha-2) h^{3+\alpha}+o\left(h^{4+\alpha}\right) \\
& =T_{1}\left(t_{i}\right) h^{2+\alpha}+O\left(h^{3+\alpha}\right), \\
E_{2, i}= & {\left.\left[k_{2}\left(t_{i}, s\right) u(s)\right]^{\prime}\right|_{s=0} \zeta(-\gamma-1) h^{2+\gamma} } \\
& +\frac{\left.\left[k_{2}\left(t_{i}, s\right) u(s)\right]^{\prime}\right|_{s=0}}{2 !} \zeta(-\gamma-2) h^{3+\gamma}+o\left(h^{4+\gamma}\right) \\
& =T_{2}\left(t_{i}\right) h^{2+\gamma}+O\left(h^{3+\gamma}\right), \\
E_{3, i} & =-\frac{\beta(1-\beta)}{2} h^{2} u^{\prime \prime}\left(q t_{i}\right)\left(q t_{i}\right)^{\gamma} k_{2}\left(t_{i}, q t_{i}\right) \\
& \cdot\left(\beta_{i} u\left(t_{[q i]}\right)+\left(1-\beta_{i}\right) u\left(t_{[q i]+1}\right)\right)+\frac{\left(q t_{i}-t_{[q i]}\right)^{2}}{12} \\
& \cdot \int_{t_{[q i]}}^{q t_{i}} \frac{\partial^{2}}{\partial s^{2}}\left[k_{2}\left(t_{i}, s\right) u(s) s^{\gamma}\right] d s+O\left(h^{3}\right)=T_{3}\left(t_{i}\right)
\end{aligned}
$$




$$
\begin{aligned}
& \cdot h^{2}+\frac{\left(q t_{i}-t_{[q i]}\right)^{2}-h^{2}}{12} \\
& \cdot \int_{t_{[q i]}}^{q t_{i}} \frac{\partial^{2}}{\partial s^{2}}\left[k_{2}\left(t_{i}, s\right) u(s) s^{\gamma}\right] d s+O\left(h^{3}\right)=T_{3}\left(t_{i}\right) \\
& \cdot h^{2}+O\left(h^{3}\right),
\end{aligned}
$$

where

$$
\begin{aligned}
& T_{1}\left(t_{i}\right)=\left.\left[k_{1}\left(t_{i}, s\right) u(s)\right]^{\prime}\right|_{s=0} \zeta(-\alpha-1), \\
& T_{2}\left(t_{i}\right)=\left.\left[k_{2}\left(t_{i}, s\right) u(s)\right]^{\prime}\right|_{s=0} \zeta(-\gamma-1), \\
& T_{3}\left(t_{i}\right)=-\frac{\beta(1-\beta)}{2} u^{\prime \prime}\left(q t_{i}\right)\left(q t_{i}\right)^{\gamma} k_{2}\left(t_{i}, q t_{i}\right) \\
& \cdot\left(\beta_{i} u\left(t_{[q i]}\right)+\left(1-\beta_{i}\right) u\left(t_{[q i]+1}\right)\right)+\frac{1}{12} \\
& \cdot \int_{t_{[q i]}}^{q t_{i}} \frac{\partial^{2}}{\partial s^{2}} k_{2}\left(t_{i}, s\right) u(s) s^{\gamma} d s .
\end{aligned}
$$

The discrete forms of (1) are obtained:

$$
\begin{aligned}
& u\left(t_{0}\right)=g\left(t_{0}\right) ; \\
& u\left(t_{i}\right)=g\left(t_{i}\right)+h \sum_{k=1}^{i-1} t_{k}^{\alpha} k_{1}\left(t_{i}, t_{k}\right) u\left(t_{k}\right)+\frac{h}{2} t_{i}^{\alpha} k_{1}\left(t_{i}, t_{i}\right) \\
& \cdot u\left(t_{i}\right)-\zeta(-\alpha) h^{1+\alpha} k_{1}\left(t_{i}, t_{0}\right) u\left(t_{0}\right) \\
& +h \sum_{k=1}^{[q i]-1} t_{k}^{\gamma} k_{2}\left(t_{i}, t_{k}\right) u\left(t_{k}\right)+\frac{h}{2} t_{[q i]}^{\gamma} k_{2}\left(t_{i}, t_{[q i]}\right) \\
& \cdot u\left(t_{[q i]}\right)-\zeta(-\gamma) h^{1+\gamma} k_{2}\left(t_{i}, t_{0}\right) u\left(t_{0}\right) \\
& +\frac{q t_{i}-t_{[q i]}}{2}\left[t_{[q i]}^{\gamma} k_{2}\left(t_{i}, t_{[q i]}\right) u\left(t_{[q i]}\right)+\left(q t_{i}\right)^{\gamma}\right. \\
& \left.\cdot k_{2}\left(t_{i}, q t_{i}\right)\left(\beta_{i} u\left(t_{[q i]}\right)+\left(1-\beta_{i}\right) u\left(t_{[q i]+1}\right)\right)\right] \\
& +E_{1, i}+E_{2, i}+E_{3, i}, \quad i=1, \ldots, N .
\end{aligned}
$$

Let $u_{i}$ be the approximate solution of $u\left(t_{i}\right)$ and ignore the error function; (32) becomes

$$
\begin{aligned}
u_{0} & =g\left(t_{0}\right) ; \\
u_{i} & =g\left(t_{i}\right)+h \sum_{k=1}^{i-1} t_{k}^{\alpha} k_{1}\left(t_{i}, t_{k}\right) u_{k}+\frac{h}{2} t_{i}^{\alpha} k_{1}\left(t_{i}, t_{i}\right) u_{i} \\
& -\zeta(-\alpha) h^{1+\alpha} k_{1}\left(t_{i}, t_{0}\right) u_{0}+h \sum_{k=1}^{[q i]-1} t_{k}^{\gamma} k_{2}\left(t_{i}, t_{k}\right) u_{k} \\
& +\frac{h}{2} t_{[q i]}^{\gamma} k_{2}\left(t_{i}, t_{[q i]}\right) u_{[q i]}-\zeta(-\gamma) h^{1+\gamma} k_{2}\left(t_{i}, t_{0}\right) u_{0}
\end{aligned}
$$

$$
\begin{aligned}
& +\frac{q t_{i}-t_{[q i]}}{2}\left[t_{[q i]}^{\gamma} k_{2}\left(t_{i}, t_{[q i]}\right) u_{[q i]}\right. \\
& +\left(q t_{i}\right)^{\gamma} k_{2}\left(t_{i} \cdot q t_{i}\right)\left(\beta_{i} u_{[q i]}+\left(1-\beta_{i}\right) u_{[q i]+1}\right] .
\end{aligned}
$$

The iterative algorithm is built to solve (33).

Iterative Algorithm

Step 1. Take sufficiently small $\epsilon>0$ and set $u_{0}=g\left(t_{0}\right) ; i:=1$;

Step 2. Let $u_{i}^{0}=u_{i-1}, m:=0$; then compute $u_{i}^{m+1}(i \leq N)$ as follows:

$$
\begin{aligned}
& u_{i}^{m+1}=g\left(t_{i}\right)+h \sum_{k=1}^{i-1} t_{k}^{\alpha} k_{1}\left(t_{i}, t_{k}\right) u_{k}^{m}+\frac{h}{2} t_{i}^{\alpha} k_{1}\left(t_{i}, t_{i}\right) u_{i}^{m} \\
& -\zeta(-\alpha) h^{1+\alpha} k_{1}\left(t_{i}, t_{0}\right) u_{0}+h \sum_{k=1}^{[q i]-1} t_{k}^{\gamma} k_{2}\left(t_{i}, t_{k}\right) u_{k}^{m} \\
& +\frac{h}{2} t_{[q i]}^{\gamma} k_{2}\left(t_{i}, t_{[q i]}\right) u_{[q i]}^{m}-\zeta(-\gamma) h^{1+\gamma} k_{2}\left(t_{i}, t_{0}\right) u_{0} \\
& +\frac{q t_{i}-t_{[q i]}}{2}\left[t_{[q i]}^{\gamma} k_{2}\left(t_{i}, t_{[q i]}\right) u_{[q i]}^{m}\right. \\
& +\left(q t_{i}\right)^{\gamma} k_{2}\left(t_{i}, q t_{i}\right)\left(\beta_{i} u_{[q i]}^{m}+\left(1-\beta_{i}\right) u_{[q i]+1}^{m}\right]
\end{aligned}
$$

Step 3. If $\left|u_{i}^{m+1}-u_{i}^{m}\right| \leq \epsilon$, set $u_{i}:=u_{i}^{m+1}$ and $i=i+1$ and return to Step 2; else, let $m=m+1$ and return to Step 2 .

\section{The Existence and Uniqueness of the Solution for the Approximate Equation}

Now, we prove the existence and uniqueness of the solution for the approximate equation. We first introduce the following lemma.

Lemma 5 (see [23]). Suppose that the sequence $\left\{w_{n}\right\}, n=$ $0, \ldots, N$, satisfies

$$
\left|w_{n}\right| \leq h \sum_{k=1}^{n} B_{k}\left|w_{k}\right|+A, \quad 0 \leq n \leq N,
$$

where $A$ and $B_{k}(k=1, \ldots, N)$ are nonnegative constants. Let $h \max _{1 \leq k \leq N} B_{k} \leq 1 / 2$ with $h=1 / N$; then we can derive

$$
\max _{0 \leq n \leq N}\left|w_{n}\right| \leq A \exp \left(2 h \sum_{k=1}^{N} B_{k}\right) .
$$

Theorem 6. Assume that $h$ is sufficiently small; then the solution of (34) is existent uniquely, and the algorithm converges at a geometrical rate.

Proof. From the nature of the delay function $\theta(t)$, we discuss the existence and uniqueness of the solution for the approximate equation under two situations. 
First, we prove that the solution of (34) is existent under two situations.

(1) One situation is $[q i]+1=i$; that is, when $i<1 /(1-q)$, we can easily obtain

$$
\begin{aligned}
& \left|u_{i}^{m+1}-u_{i}^{m}\right| \leq \frac{h}{2} t_{i}^{\alpha}\left|k_{1}\left(t_{i}, t_{i}\right)\right|\left|u_{i}^{m}-u_{i}^{m-1}\right| \\
& +\frac{q t_{i}-t_{[q i]}}{2}\left(q t_{i}\right)^{\gamma}\left|k_{2}\left(t_{i}, q t_{i}\right)\right|\left(1-\beta_{i}\right) \\
& \cdot\left|u_{[q i]+1}^{m}-u_{[q i]+1}^{m-1}\right| \leq C \frac{h}{2}\left(t_{i}^{\alpha}+\left(q t_{i}\right)^{\gamma}\left(1-\beta_{i}\right)\right) \\
& \cdot\left|u_{i}^{m}-u_{i}^{m-1}\right| .
\end{aligned}
$$

Let $C(h / 2)\left(t_{i}^{\alpha}+\left(q t_{i}\right)^{\gamma}\left(1-\beta_{i}\right)\right) \leq 1 / 2$ for a sufficiently small $h$; then $\left|u_{i}^{m+1}-u_{i}^{m}\right| \leq(1 / 2)\left|u_{i}^{m}-u_{i}^{m-1}\right|$ holds.

(2) The other situation is $[q i]+1<i$; that is, when $i>$ $1 /(1-q)$, then

$$
\begin{aligned}
\left|u_{i}^{m+1}-u_{i}^{m}\right| & \leq \frac{h}{2} t_{i}^{\alpha}\left|k_{1}\left(t_{i}, t_{i}\right)\right|\left|u_{i}^{m}-u_{i}^{m-1}\right| \\
& \leq C \frac{h}{2} t_{i}^{\alpha}\left|u_{i}^{m}-u_{i}^{m-1}\right| .
\end{aligned}
$$

Let $C(h / 2) t_{i}^{\alpha} \leq 1 / 2$ for a sufficiently small $h$; then $\mid u_{i}^{m+1}-$ $u_{i}^{m}|\leq(1 / 2)| u_{i}^{m}-u_{i}^{m-1} \mid$ holds.

With the discussion of the above two situations, one can conclude that the iterative algorithm is convergent geometrically, and the limit is the solution of (34); therefore, the solution of (34) is existent.

Next, we prove that the solution of (34) is unique. If $u_{i}$ and $v_{i}$ are solutions of (34), the difference can be represented as $\left|z_{i}\right|=\left|u_{i}-v_{i}\right|, 1 \leq i \leq N$, and

$$
\begin{aligned}
& \left|z_{0}\right|=0 ; \\
& \left|z_{i}\right|=\sum_{j=1}^{i-1} h t_{j}^{\alpha}\left|k_{1}\left(t_{i}, t_{j}\right)\right|\left|z_{j}\right|+\frac{h}{2} t_{i}^{\alpha}\left|k_{1}\left(t_{i}, t_{i}\right)\right|\left|z_{i}\right| \\
& \quad-\zeta(-\alpha) h^{1+\alpha}\left|k_{1}\left(t_{i}, t_{0}\right)\right|\left|z_{0}\right| \\
& \quad+\sum_{j=1}^{[q i]-1} h t_{j}^{\gamma}\left|k_{2}\left(t_{i}, t_{j}\right)\right|\left|z_{j}\right|+\frac{h}{2} t_{[q i]}^{\gamma}\left|k_{2}\left(t_{i}, t_{[q i]}\right)\right| \\
& \quad \cdot\left|z_{[q i]}\right|-\zeta(-\gamma) h^{1+\gamma}\left|k_{2}\left(t_{i}, t_{0}\right)\right|\left|z_{0}\right| \\
& \quad+\frac{q t_{i}-t_{[q i]}\left[t_{[q i]}^{\gamma}\left|k_{2}\left(t_{\mathrm{i}}, t_{[q i]}\right)\right|\left|z_{[q i]}\right|\right.}{2} \\
& \quad+\left(q t_{i}\right)^{\gamma} k_{2}\left(t_{i}, q t_{i}\right)\left(\beta_{i}\left|z_{[q i]}\right|+\left(1-\beta_{i}\right)\left|z_{[q i]+1}\right|\right],
\end{aligned}
$$

and $\left|k_{i}(t, s)\right| \leq C(i=1,2)$, because $k_{i}(t, s)$ are continuous on bounded domains, with $q t_{i}-t_{[q i]} \leq h$; then

$$
\begin{aligned}
& \left|z_{0}\right|=0 \\
& \left|z_{i}\right| \leq C \sum_{j=1}^{i-1} h t_{j}^{\alpha}\left|z_{j}\right|+C \frac{h}{2} t_{i}^{\alpha}\left|z_{i}\right|+\sum_{j=1}^{[q i]-1} h t_{j}^{\gamma} C\left|z_{j}\right|+\frac{h}{2}
\end{aligned}
$$

$$
\begin{aligned}
& \cdot t_{[q i]}^{\gamma} C\left|z_{[q i]}\right|+C \frac{q t_{i}-t_{[q i]}}{2}\left[t_{[q i]}^{\gamma}\left|z_{[q i]}\right|\right. \\
& +\left(q t_{i}\right)^{\gamma}\left(\beta_{i}\left|z_{[q i]}\right|+\left(1-\beta_{i}\right)\left|z_{[q i]+1}\right|\right] \\
& \leq C \sum_{j=1}^{i-1} h t_{j}^{\alpha}\left|z_{j}\right|+C \frac{h}{2} t_{i}^{\alpha}\left|z_{i}\right|+\sum_{j=1}^{[q i]-1} h t_{j}^{\gamma} C\left|z_{j}\right|+\frac{h}{2} \\
& \cdot t_{[q i]}^{\gamma} C\left|z_{[q i]}\right|+C \frac{h}{2}\left[t_{[q i]}^{\gamma}\left|z_{[q i]}\right|+t_{[q i]}^{\gamma}\left(\beta_{i}\left|z_{[q i]}\right|\right.\right. \\
& \left.+\left(1-\beta_{i}\right)\left|z_{[q i]+1}\right|\right]
\end{aligned}
$$

with $t_{i}=i h, i=1, \ldots, N$. Let $\mu=\min \{\alpha, \gamma\}$.

(1) The first situation is $[q i]+1=i$, that is, when $i<$ $1 /(1-q)$. Let $h B_{j} \leq 1 / 2$ for a sufficiently small $h$; then (40) can be written as follows:

$$
\begin{aligned}
\left|z_{i}\right| & \leq C \sum_{j=1}^{[q i]-1} h t_{j}^{\mu}\left|z_{j}\right|+C h t_{[q i]}^{\mu}\left|z_{[q i]}\right|+C \frac{h}{2} t_{i}^{\mu}\left|z_{i}\right| \\
& +C \sum_{j=1}^{[q i]-1} h t_{j}^{\mu}\left|z_{j}\right|+C \frac{h}{2} t_{[q i]}^{\mu}\left|z_{[q i]}\right|+C \frac{h}{2}\left[t_{[q i]}^{\mu}\left|z_{[q i]}\right|\right. \\
& \left.+t_{[q i]}^{\mu}\left(\beta_{i}\left|z_{[q i]}\right|+\left(1-\beta_{i}\right)\left|z_{[q i]+1}\right|\right)\right] \\
& =2 C \sum_{j=1}^{[q i]-1} h t_{j}^{\mu}\left|z_{j}\right|+\left(2 C h t_{[q i]}^{\mu}+C \frac{h}{2} t_{[q i]}^{\mu} \beta_{i}\right)\left|z_{[q i]}\right| \\
& +\left(C \frac{h}{2} t_{i}^{\mu}+C \frac{h}{2} t_{[q i]}^{\mu}\left(1-\beta_{i}\right)\right)\left|z_{[q i]+1}\right| ;
\end{aligned}
$$

namely,

$$
\begin{aligned}
\left|z_{i}\right| \leq & \sum_{j=1}^{i} B_{j}\left|z_{j}\right| \\
= & 2 C \sum_{j=1}^{[q i]-1} h t_{j}^{\mu}\left|z_{j}\right|+\left(2 C h t_{[q i]}^{\mu}+C \frac{h}{2} t_{[q i]}^{\mu} \beta_{i}\right)\left|z_{[q i]}\right| \\
& +\left(C \frac{h}{2} t_{i}^{\mu}+C \frac{h}{2} t_{[q i]}^{\mu}\left(1-\beta_{i}\right)\right)\left|z_{[q i]+1}\right|
\end{aligned}
$$

where

$$
\begin{aligned}
& B_{j} \\
& = \begin{cases}2 C \sum_{j=1}^{[q i]-1} t_{j}^{\mu}, & j=1, \ldots,[q i]-1, \\
2 C t_{[q i]}^{\mu}+C \frac{1}{2} t_{[q i]}^{\mu} \beta_{i}, & j=[q i], \\
C \frac{1}{2} t_{i}^{\mu}+C \frac{1}{2} t_{[q i]}^{\mu}\left(1-\beta_{i}\right), & j=i .\end{cases}
\end{aligned}
$$


(2) The second situation is $[q i]+1<i$, that is, when $i>$ $1 /(1-q)$. Let $h B_{j} \leq 1 / 2$ for a sufficiently small $h$; then (40) can be written as

$$
\begin{aligned}
\left|z_{i}\right| & \leq C \sum_{j=1}^{[q i]-1} h t_{j}^{\mu}\left|z_{j}\right|+C h t_{[q i]}^{\mu}\left|z_{[q i]}\right| \\
& +C h t_{[q i]+1}^{\mu}\left|z_{[q i]+1}\right|+\sum_{j=[q i]+1}^{i-1} h t_{j}^{\mu}\left|z_{j}\right|+C \frac{h}{2} t_{i}^{\mu}\left|z_{i}\right| \\
& +C \sum_{j=1}^{[q i]-1} h t_{j}^{\mu}\left|z_{j}\right|+C \frac{h}{2} t_{[q i]}^{\mu}\left|z_{[q i]}\right|+C \\
& +\frac{h}{2}\left[t_{[q i]}^{\mu}\left|z_{[q i]}\right|+t_{[q i]}^{\mu}\left(\beta_{i}\left|z_{[q i]}\right|+\left(1-\beta_{i}\right)\left|z_{[q i]+1}\right|\right]\right. \\
& =2 C h \sum_{j=1}^{[q i]-1} t_{j}^{\mu}\left|z_{j}\right|+\left(2 C h t_{[q i]}^{\mu}+C \frac{h}{2} t_{[q i]}^{\mu} \beta_{i}\right)\left|z_{[q i]}\right| \\
& \left.+\left(C h t_{[q i]+1}^{\mu}+C \frac{h}{2} t_{[q i]}^{\mu}\left(1-\beta_{i}\right)\right) \mid z_{[q i]+1}\right) \mid \\
& +\sum_{j=[q i]+1}^{i-1} h t_{j}^{\mu}\left|z_{j}\right|+C \frac{h}{2} t_{i}^{\mu}\left|z_{i}\right| ;
\end{aligned}
$$

namely,

$$
\begin{aligned}
\left|z_{i}\right| \leq & \sum_{j=1}^{i} B_{j}\left|z_{j}\right| \\
= & 2 C h \sum_{j=1}^{[q i]-1} t_{j}^{\mu}\left|z_{j}\right|+\left(2 C h t_{[q i]}^{\mu}+C \frac{h}{2} t_{[q i]}^{\mu} \beta_{i}\right)\left|z_{[q i]}\right| \\
& +\left(C h t_{[q i]+1}^{\mu}+C \frac{h}{2} t_{[q i]}^{\mu}\left(1-\beta_{i}\right)\right)\left|z_{[q i]+1}\right| \\
& +\sum_{j=[q i]+1}^{i-1} h t_{j}^{\mu}\left|z_{j}\right|+C \frac{h}{2} t_{i}^{\mu}\left|z_{i}\right| .
\end{aligned}
$$

And

$$
\begin{array}{ll}
B_{j} & \begin{cases}2 C \sum_{j=1}^{[q i]-1} t_{j}^{\mu}, & j=1, \ldots,[q i]-1, \\
2 C t_{[q i]}^{\mu}+C \frac{1}{2} t_{[q i]}^{\mu} \beta_{i}, & j=[q i], \\
C t_{[q i]+1}^{\mu}+C \frac{1}{2} t_{[q i]}^{\mu}\left(1-\beta_{i}\right), & j=[q i]+1, \\
\sum_{j=[q i]+1}^{i-1} t_{j}^{\mu}, & j=[q i]+1, \ldots, i-1, \\
C \frac{1}{2} t_{i}^{\mu,} & j=i .\end{cases}
\end{array}
$$

Based on Lemma $5,\left|z_{i}\right|=0$ with $A=0$ and the solution of the discrete equation (34) is unique. The proof of Theorem 6 is completed.

\section{The Error Estimation}

In this section, we give the error estimate between the approximation solution and the exact solution of (1).

Theorem 7. Let $u(t)$ be the exact solution of (1); the kernel functions $K_{1}(t, s)=s^{\alpha} k_{1}(t, s), K_{2}(t, s)=s^{\gamma} k_{2}(t, s),-1<$ $\alpha, \gamma \leq 0$, and the functions $k_{1}(t, s)$ and $k_{2}(t, s)$ are continuous in the domains $D:=\{(t, s): 0 \leq s \leq t \leq T\}$ and $D_{\theta}:=$ $\{(t, s): 0 \leq s \leq \theta(t), t \in I\}$, respectively. Then there is a positive constant $b$ independent of $h$ such that $\left|k_{i}(t, s)\right| \leq C$ and $\left|e_{i}\right|=\left|u\left(t_{i}\right)-u_{i}\right|(i=0, \ldots, N)$ have the following estimation:

$$
\max _{1 \leq i \leq N}\left|e_{i}\right| \leq b h^{2+\mu} .
$$

Proof. From (26) and (27), we have

$$
\begin{aligned}
E_{1, i}= & \frac{\left.\left[k_{1}\left(t_{i}, s\right) u(s)\right]^{\prime}\right|_{s=0}}{1 !} \zeta(-\alpha-1) h^{2+\alpha} \\
& +\frac{\left.\left[k_{1}\left(t_{i}, s\right) u(s)\right]^{\prime}\right|_{s=0}}{2 !} \zeta(-\alpha-2) h^{3+\alpha} \\
& +o\left(h^{4+\alpha}\right)=T_{1}\left(t_{i}\right) h^{2+\alpha}+O\left(h^{3+\alpha}\right), \\
E_{2, i}= & \frac{\left.\left[k_{2}\left(t_{i}, s\right) u(s)\right]^{\prime}\right|_{s=0}}{1 !} \zeta(-\gamma-1) h^{2+\gamma} \\
& +\frac{\left.\left[k_{2}\left(t_{i}, s\right) u(s)\right]^{\prime}\right|_{s=0}}{2 !} \zeta(-\gamma-2) h^{3+\gamma} \\
& +o\left(h^{4+\gamma}\right)=T_{2}\left(t_{i}\right) h^{2+\gamma}+O\left(h^{3+\gamma}\right) .
\end{aligned}
$$

By the trapezoidal formula and Lemma 4, we have

$$
\begin{aligned}
& E_{3, i}=-\frac{\beta(1-\beta)}{2} h^{2} u^{\prime \prime}\left(q t_{i}\right)\left(q t_{i}\right)^{\gamma} k_{2}\left(t_{i}, q t_{i}\right) \\
& \cdot\left(\beta_{i} u_{t_{[q i]}}+\left(1-\beta_{i}\right) u_{t_{[q i]+1}}\right)+\frac{\left(q t_{i}-t_{[q i]}\right)^{2}}{12} \\
& \cdot \int_{t_{[q i]}}^{q t_{i}} \frac{\partial^{2}}{\partial s^{2}} k_{2}\left(t_{i}, s\right) u(s) s^{\gamma} d s+O\left(h^{3}\right)=T_{3}\left(t_{i}\right) h^{2} \\
&+\frac{\left(q t_{i}-t_{[q i]}\right)^{2}-h^{2}}{12} \int_{t_{[q i]}}^{q t_{i}} \frac{\partial^{2}}{\partial s^{2}} k_{2}\left(t_{i}, s\right) u(s) s^{\gamma} d s \\
&+O\left(h^{3}\right)=T_{3}\left(t_{i}\right) h^{2}+O\left(h^{3}\right)
\end{aligned}
$$

where

$$
\begin{aligned}
T_{3}\left(t_{i}\right)= & -\frac{\beta(1-\beta)}{2} u^{\prime \prime}\left(q t_{i}\right)\left(q t_{i}\right)^{\gamma} k_{2}\left(t_{i}, q t_{i}\right) \\
& \cdot\left(\beta_{i} u_{t_{[q i]}}+\left(1-\beta_{i}\right) u_{t_{[q i]+1}}\right)+\frac{1}{12} \\
& \cdot \int_{t_{[q i]}}^{q t_{i}} \frac{\partial^{2}}{\partial s^{2}} k_{2}\left(t_{i}, s\right) u(s) s^{\gamma} d s .
\end{aligned}
$$


Then, we have

$$
\begin{aligned}
& u\left(t_{i}\right)=g\left(t_{i}\right)+Q_{N_{1}}+Q_{N_{2}}+Q_{N_{3}}+T_{1}\left(t_{i}\right) h^{2+\alpha} \\
& +T_{2}\left(t_{i}\right) h^{2+\gamma}+T_{3}\left(t_{i}\right) h^{2}+O\left(h^{2}\right), \\
& Q_{N_{1}}=\sum_{j=1}^{i-1} h t_{j}^{\alpha} k_{1}\left(t_{i}, t j\right) u_{j}+\frac{h}{2} t_{i}^{\alpha} k_{1}\left(t_{i}, t_{i}\right) u_{i}-\zeta(-\alpha) \\
& \cdot h^{1+\alpha} u_{0} k\left(t_{i}, t_{0}\right), \\
& Q_{N_{2}}=\sum_{j=1}^{[q i]-1} h t_{j}^{\gamma} k_{2}\left(t_{i}, t j\right) u_{j}+\frac{h}{2} t_{[q i]}^{\gamma} k_{2}\left(t_{i}, t_{[q i]}\right) u_{[q i]} \\
& -\zeta(-\gamma) h^{1+\gamma} u_{0} k_{2}\left(t_{i}, t_{0}\right), \\
& Q_{N_{3}}=\frac{q t_{i}-t_{[q i]}}{2}\left[t_{[q i]}^{\gamma} k_{2}\left(t_{i}, t_{[q i]}\right) u_{[q i]}\right. \\
& +\left(q t_{i}\right)^{\gamma} k_{2}\left(t_{i}, q t_{i}\right)\left(\beta_{i} u_{[q i]}+\left(1-\beta_{i}\right) u_{[q i]+1}\right],
\end{aligned}
$$

where $\beta_{i}=1+[q i]-q i$. Subtracting (34) from (51), we get

$$
\begin{aligned}
\left|e_{0}\right| & =0, \\
\left|e_{i}\right| & =\sum_{j=1}^{i-1} h t_{j}^{\alpha}\left|k_{1}\left(t_{i}, t_{j}\right)\right|\left|e_{j}\right|+\frac{h}{2} t_{i}^{\alpha}\left|k_{1}\left(t_{i}, t_{i}\right)\right|\left|e_{i}\right| \\
& +\sum_{j=1}^{[q i]-1} h t_{j}^{\gamma}\left|k_{2}\left(t_{i}, t j\right)\right|\left|e_{j}\right|+\frac{h}{2} t_{[q i]}^{\gamma}\left|k_{2}\left(t_{i}, t[q i]\right)\right| \\
& +\left|e_{[q i]}\right|+\frac{q t_{i}-t_{[q i]}}{2}\left[t_{[q i]}^{\gamma}\left|k_{2}\left(t_{i}, t_{[q i]}\right)\right| e_{[q i]}\right. \\
& +\left(q t_{i}\right)^{\gamma}\left|k_{2}\left(t_{i}, q t_{i}\right)\right|\left(\beta_{i} e_{[q i]}+\left(1-\beta_{i}\right) e_{[q i]+1}\right] \\
& +T_{1}\left(t_{i}\right) h^{2+\alpha}+T_{2}\left(t_{i}\right) h^{2+\beta}+T_{3}\left(t_{i}\right) h^{2}+O\left(h^{3+\mu}\right) \\
& \leq C \sum_{j=1}^{i-1} h t_{j}^{\alpha}\left|e_{j}\right|+C \frac{h}{2} t_{i}^{\alpha}\left|e_{i}\right|+\sum_{j=1}^{[q i]-1} h t_{j}^{\gamma} C\left|e_{j}\right|+\frac{h}{2} \\
& +t_{[q i]}^{\gamma} C\left|e_{[q i]}\right|+C \frac{q t_{i}-t_{[q i]}}{2}\left[t_{[q i]}^{\gamma} e_{[q i]}\right. \\
& +\left(q t_{i}\right)^{\gamma}\left(\beta_{i} e_{[q i]}+\left(1-\beta_{i}\right) e_{[q i]+1}\right]+T_{1}\left(t_{i}\right) h^{2+\alpha} \\
& \left.+t_{i}\right) h^{2+\gamma}+T_{3}\left(t_{i}\right) h^{2}+O\left(h^{3+\mu}\right) .
\end{aligned}
$$

Let

$A$

$$
\begin{aligned}
& =\left|T_{1}\left(t_{i}\right) h^{2+\alpha}+T_{2}\left(t_{i}\right) h^{2+\gamma}+T_{3}\left(t_{i}\right) h^{2}+O\left(h^{3+\mu}\right)\right| \\
& =O\left(h^{2+\mu}\right)
\end{aligned}
$$

and the analysis is the same as (41) and (44); we have

$$
\left|e_{i}\right| \leq A+\sum_{j=1}^{i} B_{j}\left|e_{j}\right|, \quad 1 \leq i \leq N .
$$

From Lemma 5, there is a positive constant $b$ independent of $h$ such that

$$
\max _{1 \leq i \leq N}\left|e_{i}\right| \leq b h^{2+\mu}
$$

The proof of Theorem 7 is completed.

\section{Error Asymptotic Expansion and Extrapolation Algorithm}

In this section, we present the main theoretical result of the error asymptotic expansions and the relevant extrapolation algorithm.

Theorem 8. Based on the conditions of Theorem 7, there exist continue functions $\widehat{T}_{i}(t),(i=1,2,3)$ satisfying the asymptotic expansion

$$
\begin{aligned}
u_{i}= & \left.u\left(t_{i}\right)+\widehat{T}_{1}\left(t_{i}\right) h^{2+\alpha}+\widehat{T}_{2}\left(t_{i}\right)\right) h^{2+\gamma}+\widehat{T}_{3}\left(t_{i}\right) h^{2} \\
& +O\left(h^{3+\mu}\right), \quad-1<\alpha<0,-1<\gamma \leq 0 .
\end{aligned}
$$

Proof. Suppose that $\left\{\widehat{T}_{k}(t), k=1,2,3\right\}$ satisfies the auxiliary delay equations:

$$
\begin{aligned}
\widehat{T}_{k}(t)= & T_{k}(t)+\int_{0}^{t} s^{\alpha} k_{1}(t, s) u(s) \widehat{T}_{k}(s) d s \\
& +\int_{0}^{q t} s^{\gamma} k_{2}(t, s) u(s) \widehat{T}_{k}(s) d s,
\end{aligned}
$$

and $\widehat{T}_{k}\left(t_{i}\right), i=1, \ldots, N$, satisfy the approximation equations:

$$
\begin{aligned}
& \widehat{T}_{k}\left(t_{i}\right)=T_{k}\left(t_{i}\right)+\sum_{j=1}^{i-1} h t_{j}^{\alpha} k_{1}\left(t_{i}, t j\right) u_{j} \widehat{T}_{k}\left(t_{j}\right)+\frac{h}{2} \\
& \cdot t_{i}^{\alpha} k_{1}\left(t_{i}, t_{i}\right) u_{i} \widehat{T}_{k}\left(t_{i}\right)-\zeta(-\alpha) h^{1+\alpha} u_{0} k\left(t_{i}, t_{0}\right) \\
& \cdot \widehat{T}_{k}\left(t_{0}\right)+\sum_{j=1}^{[q i]-1} h t_{j}^{\gamma} k_{2}\left(t_{i}, t_{j}\right) u_{j} \widehat{T}_{k}\left(t_{j}\right)+\widehat{T}_{k}\left(t_{j}\right) \frac{h}{2} \\
& \cdot t_{[q i]}^{\gamma} k_{2}\left(t_{i}, t_{[q i]}\right) u_{[q i]} \widehat{T}_{k}\left(t_{q i}\right)-\zeta(-\gamma) \\
& \cdot h^{1+\gamma} u_{0} k_{2}\left(t_{i}, t_{0}\right) \widehat{T}_{k}\left(t_{0}\right) \\
& +\frac{q t_{i}-t_{[q i]}}{2}\left[t_{[q i]}^{\gamma} k_{2}\left(t_{i}, t_{[q i]}\right) u_{[q i]} \widehat{T}_{[q i]}+\left(q t_{i}\right)^{\gamma}\right. \\
& \left.\cdot k_{2}\left(t_{i}, q t_{i}\right)\left(\beta u_{q t_{i}} \widehat{T}_{[q i]}+(1-\beta) u_{[q i]+1} \widehat{T}_{[q i]+1}\right)\right] .
\end{aligned}
$$

Similar to the proof of Theorem 7, we obtain

$$
\max _{1 \leq i \leq N}\left|\widehat{T}_{k}\left(t_{i}\right)-T\left(t_{i}\right)\right| \leq C h^{2+\mu} .
$$


Let

$$
E_{i}=e_{i}-\left(T_{1}\left(t_{i}\right) h^{2+\alpha}+T_{2}\left(t_{i}\right) h^{2+\gamma}+T_{3}\left(t_{i}\right)\right) h^{2} .
$$

We have

$$
\begin{aligned}
E_{i} & =\sum_{j=1}^{i-1} h t_{j}^{\alpha} k_{1}\left(t_{i}, t_{j}\right) u_{j} E_{j}+\frac{h}{2} t_{i}^{\alpha} k_{1}\left(t_{i}, t_{i}\right) u_{i} E_{i} \\
& -\zeta(-\alpha) h^{1+\alpha} u_{0} k\left(t_{i}, t_{0}\right) E_{0}+\sum_{j=1}^{[q i]-1} h t_{j}^{\gamma} k_{2}\left(t_{i}, t_{j}\right) u_{j} E_{j} \\
& +\frac{h}{2} t_{[q i]}^{\gamma} k_{2}\left(t_{i}, t_{[q i]}\right) u_{[q i]} E_{[q i]}-\zeta(-\gamma) \\
& \cdot h^{1+\gamma} u_{0} k_{2}\left(t_{i}, t_{0}\right) E_{0} \\
& +\frac{q t_{i}-t_{[q i]}}{2}\left[t_{[q i]}^{\gamma} k_{2}\left(t_{i}, t_{[q i]}\right) u_{[q i]} \widehat{T}_{[q i]}\right. \\
& \left.+\left(q t_{i}\right)^{\gamma} k_{2}\left(t_{i}, q t_{i}\right)\left(\beta u_{q t_{i}}+(1-\beta) u_{[q i]+1}\right)\right]\left(\beta E_{[q i]}\right. \\
& \left.+(1-\beta) E_{[q i]+1}\right) .
\end{aligned}
$$

From Lemma 1, there exists a constant $d$ such that

$$
\max _{1 \leq i \leq N}\left|E_{i}\right| \leq d h^{3+\mu}
$$

The asymptotic expansion is (56).

Based on Theorem 8, we adopt the Richardson extrapolation to improve the accuracy.

\section{Extrapolation Algorithm}

Step 1. Assume that $\mu=\min (\alpha, \gamma)=\alpha$, and halve the step length to obtain

$$
\begin{aligned}
u_{i}^{h / 2}= & \left.u\left(t_{i}\right)+\widehat{T}_{1}\left(t_{i}\right)\left(\frac{h}{2}\right)^{2+\alpha}+\widehat{T}_{2}\left(t_{i}\right)\right)\left(\frac{h}{2}\right)^{2+\gamma} \\
& +\widehat{T}_{3}\left(t_{i}\right)\left(\frac{h}{2}\right)^{2}+O\left(\left(\frac{h}{2}\right)^{3+\alpha}\right) .
\end{aligned}
$$

By combining (56) with (63), we get

$$
\begin{aligned}
u_{i}^{1, h} & =\frac{2^{2+\alpha} u_{i}^{h / 2}-u_{i}^{h}}{2^{2+\alpha}-1} \\
& \left.=u\left(t_{i}\right)+\widehat{T}_{2}\left(t_{i}\right)\right) h^{2+\gamma}+\widehat{T}_{3}\left(t_{i}\right) h^{2}+O\left(h^{3+\alpha}\right) .
\end{aligned}
$$

Step 2. We implement Richardson $h^{2+\gamma}$ extrapolation:

$$
\begin{aligned}
u_{i}^{1, h / 2}= & \left.u\left(t_{i}\right)+\widehat{T}_{2}\left(t_{i}\right)\right)\left(\frac{h}{2}\right)^{2+\gamma}+\widehat{T}_{3}\left(t_{i}\right)\left(\frac{h}{2}\right)^{2} \\
& +O\left(\left(\frac{h}{2}\right)^{3+\alpha}\right) .
\end{aligned}
$$

Combining (64) with (65), we get

$$
\begin{aligned}
u_{i}^{2, h} & =\frac{2^{2+\gamma} u_{i}^{1, h / 2}-u_{i}^{1, h}}{2^{2+\gamma}-1} \\
& =u\left(t_{i}\right)+\widehat{T}_{3}\left(t_{i}\right) h^{2}+O\left(h^{3+\alpha}\right) .
\end{aligned}
$$

Moreover, a posterior asymptotic error estimate

$$
\begin{aligned}
\left|u_{i}^{h / 2}-u_{i}\right|= & \left|\frac{2^{2+\alpha} u_{i}^{h / 2}-u_{i}^{h}}{2^{2+\alpha}-1}-u(i)+\frac{u_{i}^{h}-u_{i}^{h / 2}}{2^{2+\alpha}-1}\right| \\
\leq & \left|\frac{2^{2+\alpha} u_{i}^{h / 2}-u_{i}^{h}}{2^{2+\alpha}-1}-u(i)\right|+\left|\frac{u_{i}^{h}-u_{i}^{h / 2}}{2^{2+\alpha}-1}\right| \\
= & \left|\frac{2^{2+\alpha} u_{i}^{h / 2}-u_{i}^{h}}{2^{2+\alpha}-1}-u(i)\right|+\left|\frac{u_{i}^{h}-u_{i}^{h / 2}}{2^{2+\alpha}-1}\right| \\
& +O\left(h^{2}\right) .
\end{aligned}
$$

The error $u_{i}^{h / 2}-u(i)$ is bounded by $\left(u_{i}^{h}-u_{i}^{h / 2}\right) /\left(2^{2+\alpha}-1\right)$, which is essential to construct adaptable algorithms.

\section{Numerical Experiments}

In this section, three examples will be presented to show the efficiency of the quadrature method. We design a set of grids on the interval $I$; the absolute error is denoted by

$$
e_{h}\left(t_{\mathrm{i}}\right)=\left|u\left(t_{i}\right)-u_{i}\right|
$$

with $i=1, \ldots, N ; u\left(t_{i}\right)$ and $u_{i}$ are the exact solution and the approximate solution at $t=t_{i}$, respectively. Set $h=1 / N$, and the convergence order is defined by

$$
\text { Rate }=\frac{\log \left(e_{h} / e_{h / 2}\right)}{\log 2} .
$$

Example 1. Consider the following equation:

$$
u(t)=g(t)-\int_{0}^{t}(t-s)^{\alpha} u(s) d s, \quad t \in[0, T],
$$

where $T=1,-1<\alpha<0$, and the analytical solution is $u(t)=\cos (t)$.

The numerical results at the point $t=1$ with the partitions $N=2^{4}, 2^{5}, 2^{6}, 2^{7}, 2^{8}$ are addressed in Table 1 . By the mechanical quadrature method and iterative method, the obtained absolute errors $e_{h}$ with more refined partitions show a more accurate approximate solution, and the convergent rate is adjacent to $(3 / 2)$-order. Based on the Richardson $h^{2+\alpha}$ extrapolation, the errors are closer to the exact solution, and the convergence order is improved to 2 -order. The posteriori error is also achieved. The two kinds of convergence orders are consistent with the theoretical analysis. 
TABLE 1: Numerical results at point $t=1$ of Example 1.

\begin{tabular}{|c|c|c|c|c|c|}
\hline $\mathrm{N}$ & $e_{h}$ & Rate & $\mathrm{R}-h^{2+\alpha}$ extrapolation & Rate & Posteriori errors \\
\hline $2^{4}$ & $9.27 \mathrm{e}-04$ & - & - & - & - \\
\hline $2^{5}$ & $3.22 \mathrm{e}-04$ & $2^{1.53}$ & $9.65 e-06$ & - & $3.31 \mathrm{e}-04$ \\
\hline $2^{6}$ & $1.12 \mathrm{e}-04$ & $2^{1.52}$ & $2.24 \mathrm{e}-06$ & $2^{2.10}$ & $1.14 \mathrm{e}-04$ \\
\hline $2^{7}$ & $3.93 e-05$ & $2^{1.51}$ & $5.32 \mathrm{e}-07$ & $2^{2.08}$ & $3.99 \mathrm{e}-05$ \\
\hline $2^{8}$ & $1.38 \mathrm{e}-05$ & $2^{1.51}$ & $1.28 \mathrm{e}-07$ & $2^{2.06}$ & $1.39 \mathrm{e}-05$ \\
\hline
\end{tabular}

TABLE 2: Numerical results of Example 2.

\begin{tabular}{lcccc}
\hline $\mathrm{t}$ & $e_{h}$ & $e_{h / 2}$ & $e_{h / 4}$ & Posteriori error \\
\hline 0.2 & $3.89 \mathrm{e}-06$ & $7.84 \mathrm{e}-07$ & $1.77 \mathrm{e}-07$ & $1.69 \mathrm{e}-06$ \\
0.4 & $5.05 \mathrm{e}-06$ & $1.01 \mathrm{e}-06$ & $2.28 \mathrm{e}-07$ & $2.20 \mathrm{e}-06$ \\
0.6 & $5.67 \mathrm{e}-06$ & $1.14 \mathrm{e}-06$ & $2.55 \mathrm{e}-07$ & $2.48 \mathrm{e}-06$ \\
0.8 & $5.98 \mathrm{e}-06$ & $1.19 \mathrm{e}-06$ & $2.68 \mathrm{e}-07$ & $2.61 \mathrm{e}-06$ \\
1.0 & $6.07 \mathrm{e}-06$ & $1.21 \mathrm{e}-06$ & $2.71 \mathrm{e}-07$ & $2.66 \mathrm{e}-06$ \\
\hline
\end{tabular}

TABLE 3: Numerical results of Example 3.

\begin{tabular}{lcccc}
\hline $\mathrm{t}$ & $e_{h}$ & $e_{h / 2}$ & $e_{h / 4}$ & Posteriori errors \\
\hline 0.2 & $1.43 \mathrm{e}-06$ & $9.29 \mathrm{e}-07$ & $7.18 \mathrm{e}-07$ & $2.75 \mathrm{e}-07$ \\
0.4 & $1.23 \mathrm{e}-05$ & $4.52 \mathrm{e}-07$ & $9.09 \mathrm{e}-07$ & $6.48 \mathrm{e}-06$ \\
0.6 & $4.79 \mathrm{e}-05$ & $8.27 \mathrm{e}-06$ & $5.23 \mathrm{e}-07$ & $2.17 \mathrm{e}-05$ \\
0.8 & $1.34 \mathrm{e}-04$ & $2.90 \mathrm{e}-05$ & $5.10 \mathrm{e}-06$ & $5.74 \mathrm{e}-05$ \\
1.0 & $3.25 \mathrm{e}-04$ & $7.63 \mathrm{e}-05$ & $1.16 \mathrm{e}-05$ & $1.36 \mathrm{e}-04$ \\
\hline
\end{tabular}

Example 2. Consider the following delay Volterra integral equation with weakly singular kernel as

$$
\begin{array}{r}
u(t)=g(t)-\int_{0}^{t} s^{-1 / 2} u(s) d s+\int_{0}^{q t} s^{-1 / 2} u(s) d s, \\
t \in[0, T],
\end{array}
$$

with $q=0.9, T=1$, and the initial value $u(0)=g(0)=0$, and $g(t)$ is determined by the analytical solution $u(t)=t+1$.

We denote the approximate solution by $u_{h}$ with $h=1 / 32$. The error results at some interior points in the interval $t \in$ $[0,1]$ with different partition are listed in Table 2 . The comparison of the exact solution and the approximate solution with partition $N=2^{3}$ is shown in Figure 1. It is obvious that this paper provides a high accuracy algorithm for the weakly singular Volterra integral equation with proportional delays.

Example 3. Consider the following delay Volterra integral equation with weakly singular kernel as

$$
\begin{array}{r}
u(t)=g(t)-\int_{0}^{t} s^{-1 / 4} u(s) d s+\int_{0}^{q t}\left(t^{2}+s\right) u(s) d s, \\
t \in[0, T],
\end{array}
$$

with $q=0.9, T=1$, and the initial value $u(0)=g(0)=0$, and $g(t)$ is determined by the analytical solution $u(t)=t+1$.

The absolute error $\left|e_{h}(t)\right|=\left|u(t)-u_{h}(t)\right|, t \in[0,1]$, and the posteriori errors at some interior points are listed in Table 3 when $N=2^{5}, 2^{6}, 2^{7}$, which can observe that the error results decay quickly with the increasing of $N$. The absolute errors between the exact solution and the approximate solution with partition $N=2^{2}, 2^{3}$ are shown in Figure 2, which indicate that our algorithm is effective.

\section{Conclusion}

In this paper, we use the mechanical quadrature method and Romberg extrapolation for weakly Volterra integral equation with proportional delays. Most papers analyze the delay Volterra integral equation with continuous kernels; the study for the delay Volterra integral equation with weakly singular kernels still faces a real challenge for the relevant researchers in both numerical computation and theoretical analysis. The improved Gronwall inequality is adopted to prove the existence and uniqueness of the solution of the original equation. At the same time, the discrete Gronwall equality and iterative method are adopted to prove the existence and uniqueness of the solution of the approximate equation. Moreover, according to the error asymptotic expansion of the mechanical quadrature method and the extrapolation, a high order of accuracy can be achieved and a posterior error estimation can be obtained. Both the theoretical analysis and the numerical examples show that the presented method is efficient.

\section{Data Availability}

The data used to support the findings of this study are available from the corresponding author upon request. 


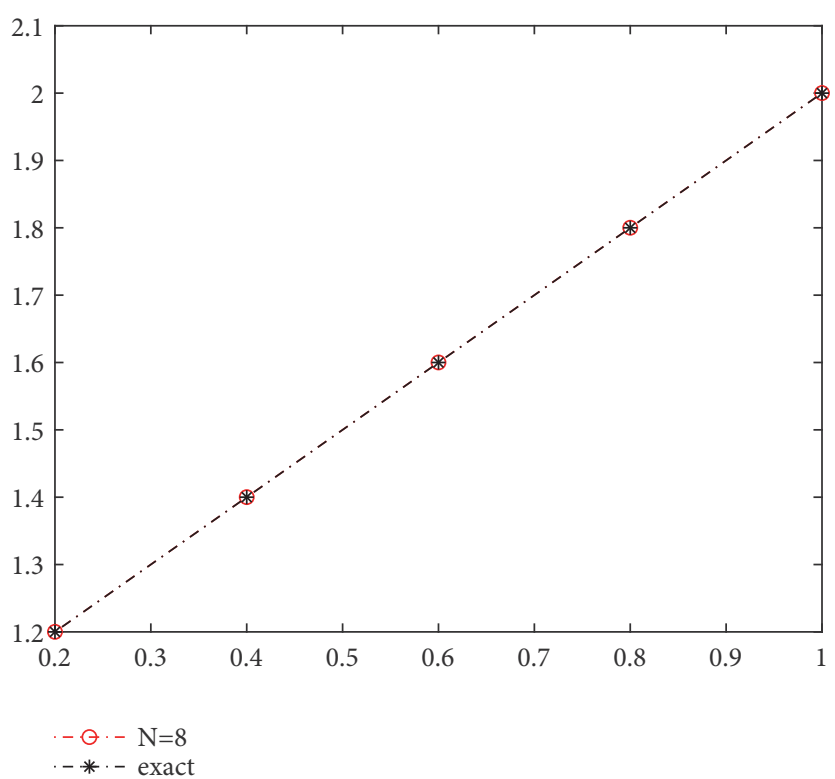

Figure 1: Comparison of exact solution and approximate solution with $N=2^{3}$.

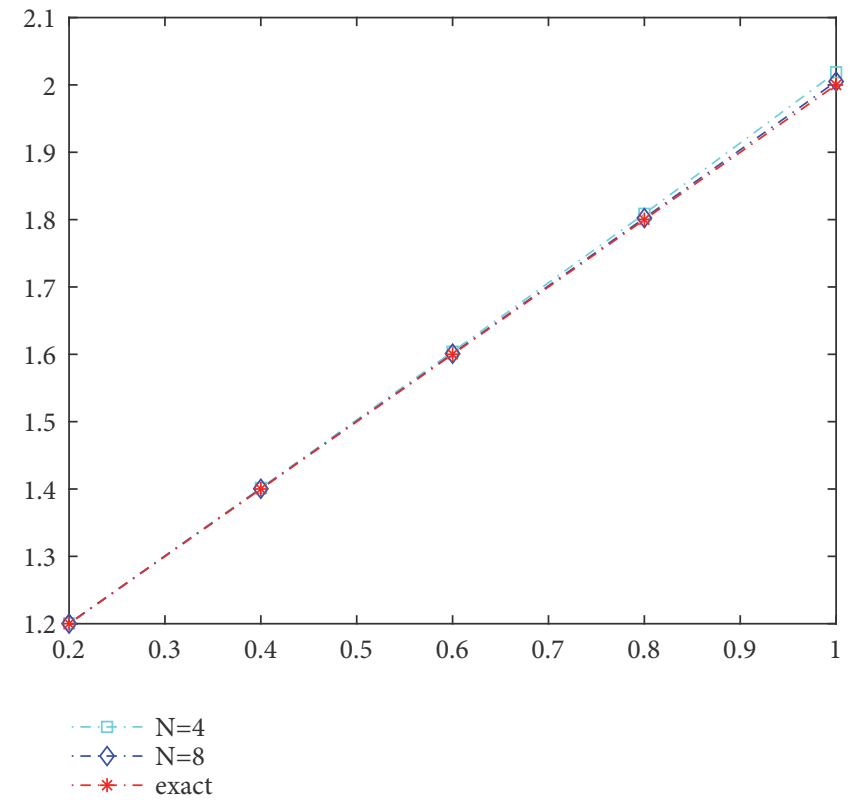

Figure 2: Comparison of exact solution and approximate solution with $N=2^{3}$.

\section{Conflicts of Interest}

The authors declare that there are no conflicts of interest regarding the publication of this article.

\section{Acknowledgments}

This work was supported by the financial support from the National Natural Science Foundation of China (Grant no. 11371079).

\section{References}

[1] F. Brauer, "Constant rate harvesting of populations governed by Volterra integral equations," Journal of Mathematical Analysis and Applications, vol. 56, no. 1, pp. 18-27, 1976.

[2] K. L. Cooke and J. L. Kaplan, "A periodicity threshold theorem for epidemics and population growth," Mathematical Biosciences, vol. 31, no. 1-2, pp. 87-104, 1976.

[3] P. K. Lamm and T. L. Scofield, "Sequential predictor-corrector methods for the variable regularization of Volterra inverse problems," Inverse Problems, vol. 16, no. 2, pp. 373-399, 2000. 
[4] H. Brunner, "Collocation methods for Volterra integral and related functional differential equations," in Mathematics of Computation, vol. 75, p. 254, Cambridge University Press, 2009.

[5] G. A. Okeke and M. Abbas, "A solution of delay differential equations via Picard-Krasnoselskii hybrid iterative process," Arabian Journal of Mathematics, vol. 6, no. 1, pp. 21-29, 2017.

[6] N. Bildik and S. Deniz, "A new efficient method for solving delay differential equations and a comparison with other methods," The European Physical Journal Plus, vol. 132, no. 1, p. 51, 2017.

[7] F. Mohammadi, "Numerical solution of systems of fractional delay differential equations using a new kind of wavelet basis," Computational \& Applied Mathematics, vol. 37, no. 4, pp. 41224144, 2018.

[8] K. Harriman, P. Houston, B. Senior, and E. Sli, "hp-version discontinuous Galerkin methods with interior penalty for partial differential equations with nonnegative characteristic form," in Recent Advances in Scientific Computing and Partial Differential Equations, vol. 330 of Contemp. Math., pp. 89-119, 2003.

[9] C.-T. Sheng, Z.-Q. Wang, and B.-Y. Guo, "An hp-spectral collocation method for nonlinear Volterra functional integrodifferential equations with delays," Applied Numerical Mathematics, vol. 105, pp. 1-24, 2016.

[10] T. Koto, "Stability of Runge-Kutta methods for delay integrodifferential equations," Journal of Computational and Applied Mathematics, vol. 145, no. 2, pp. 483-492, 2002.

[11] H. Cai, Y. Chen, and Y. Huang, "A Legendre-Petrov-Galerkin method for solving Volterra integro-differential equations with proportional delays," International Journal of Computer Mathematics, pp. 1-15, 2018.

[12] M. Mosleh and M. Otadi, "Least squares approximation method for the solution of Hammerstein-Volterra delay integral equations," Applied Mathematics and Computation, vol. 258, pp. 105110, 2015.

[13] I. Ali, H. Brunner, and T. Tang, "Spectral methods for pantograph-type differential and integral equations with multiple delays," Frontiers of Mathematics in China, vol. 4, no. 1, pp. 49-61, 2009.

[14] P. K. Sahu and S. S. Ray, "A new Bernoulli wavelet method for accurate solutions of nonlinear fuzzy Hammerstein-Volterra delay integral equations," Fuzzy Sets and Systems, vol. 309, pp. 131-144, 2017.

[15] K. Zhang and J. Li, "Collocation methods for a class of Volterra integral functional equations with multiple proportional delays," Advances in Applied Mathematics and Mechanics, vol. 4, no. 5, pp. 575-602, 2012.

[16] H. Xie, R. Zhang, and H. Brunner, "Collocation methods for general Volterra functional integral equations with vanishing delays," SIAM Journal on Scientific Computing, vol. 33, no. 6, pp. 3303-3332, 2011.

[17] P. Darania and S. Pishbin, "High-order collocation methods for nonlinear delay integral equation," Journal of Computational and Applied Mathematics, vol. 326, pp. 284-295, 2017.

[18] Z.-Q. Wang and C.-T. Sheng, "An hp-spectral collocation method for nonlinear Volterra integral equations with vanishing variable delays," Mathematics of Computation, vol. 85, no. 298, pp. 635-666, 2016.

[19] Z. Gu and Y. Chen, "Chebyshev spectral-collocation method for a class of weakly singular Volterra integral equations with proportional delay," Journal of Numerical Mathematics, vol. 22, no. 4, pp. 311-341, 2014.
[20] A. Sidi and M. Israeli, "Quadrature methods for periodic singular and weakly singular fredholm integral equations," Journal of Scientific Computing, vol. 3, no. 2, pp. 201-231, 1988.

[21] H. Brunner, "Recent advances in the numerical analysis of Volterra functional differential equations with variable delays," Journal of Computational and Applied Mathematics, vol. 228, no. 2, pp. 524-537, 2009.

[22] J. Huang, High Precision Algorithm for Multidimensional Singular Integral, Science Press, 2017.

[23] L. Tao and H. Yong, "A generalization of discrete Gronwall inequality and its application to weakly singular Volterra integral equation of the second kind," Journal of Mathematical Analysis and Applications, vol. 282, no. 1, pp. 56-62, 2003. 


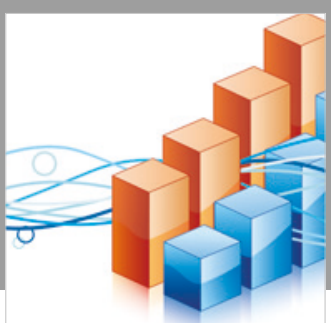

Advances in

Operations Research

\section{-n-m}
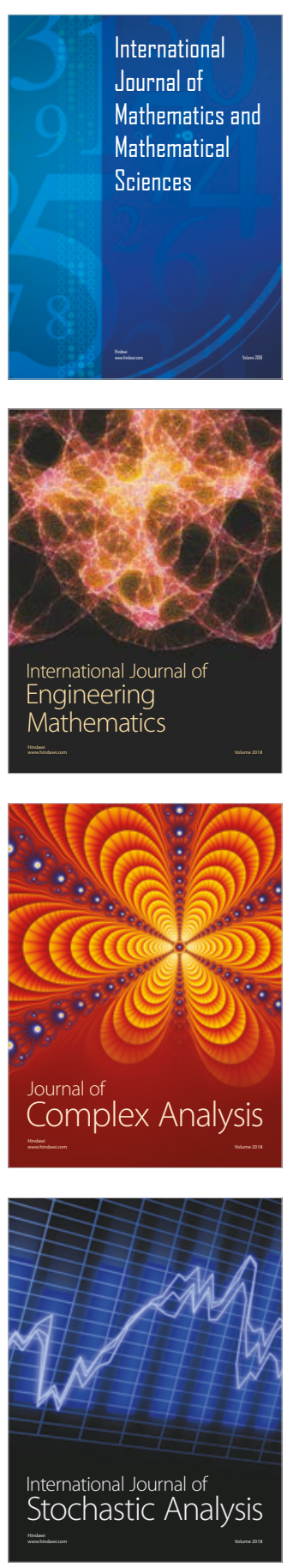
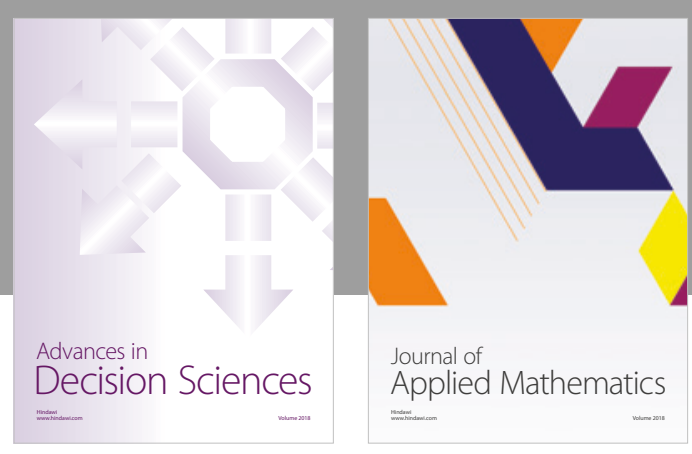

Journal of

Applied Mathematics
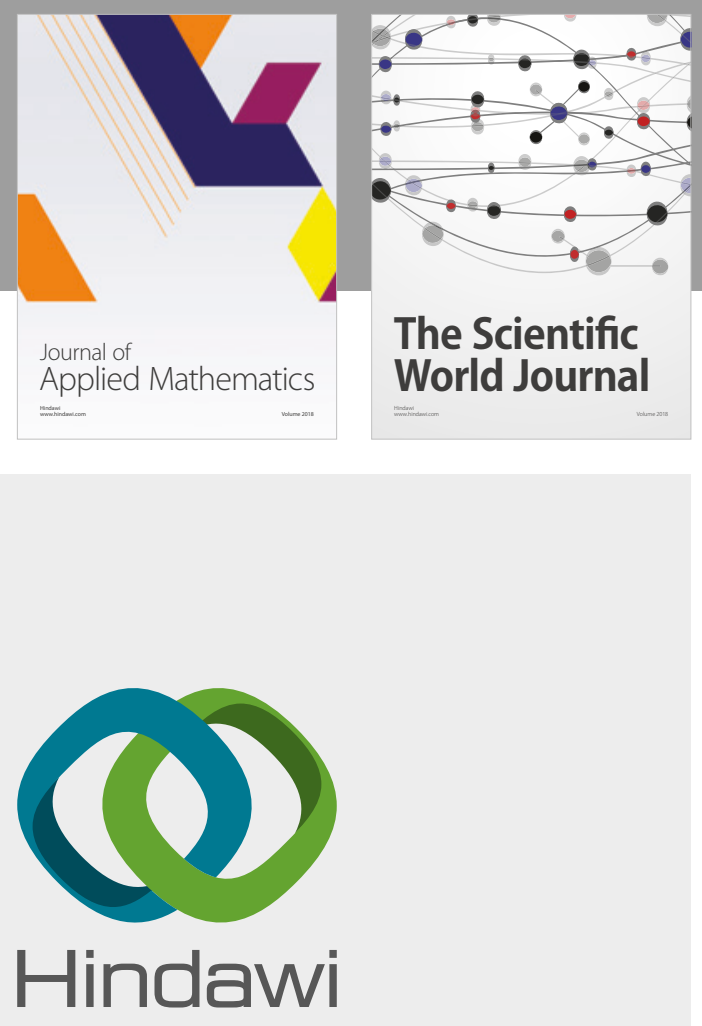

Submit your manuscripts at

www.hindawi.com

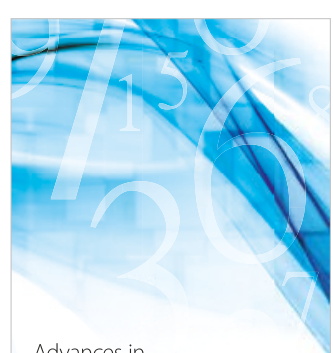

Advances in
Numerical Analysis
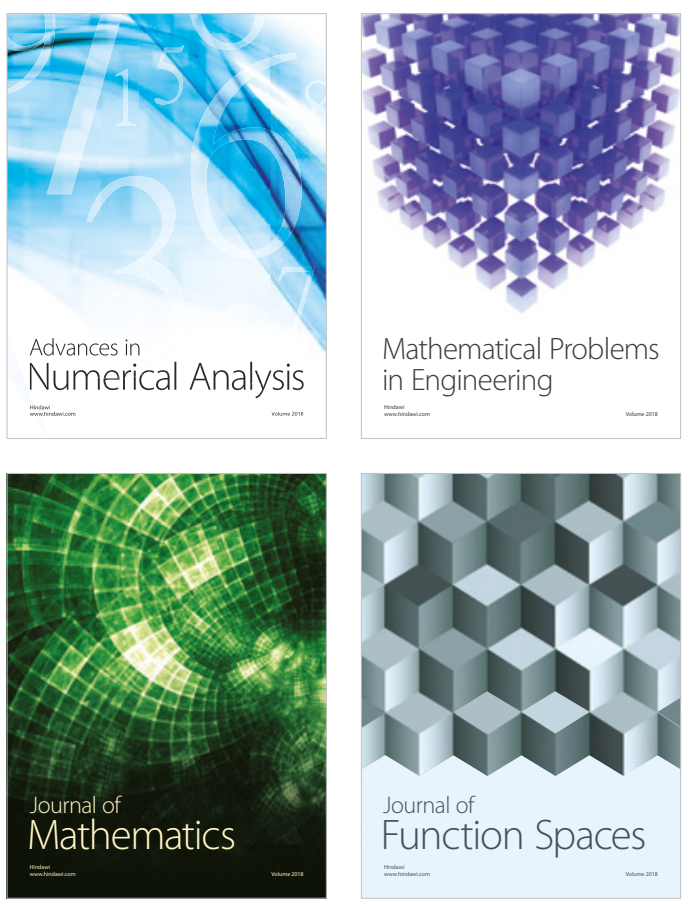

Mathematical Problems in Engineering

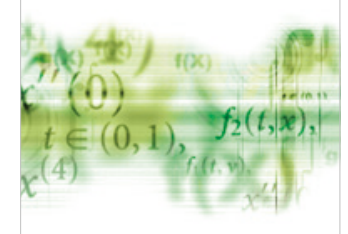

International Journal of

Differential Equations

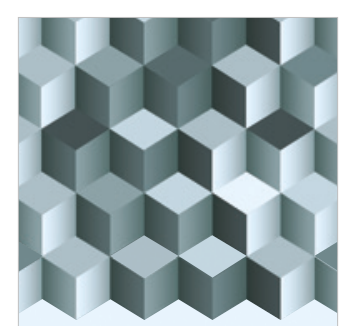

Journal of

Function Spaces
The Scientific

World Journal

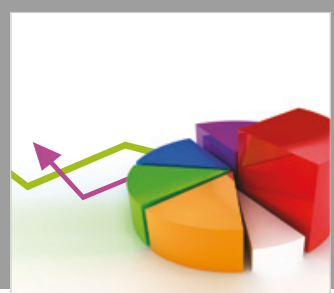

Journal of

Probability and Statistics
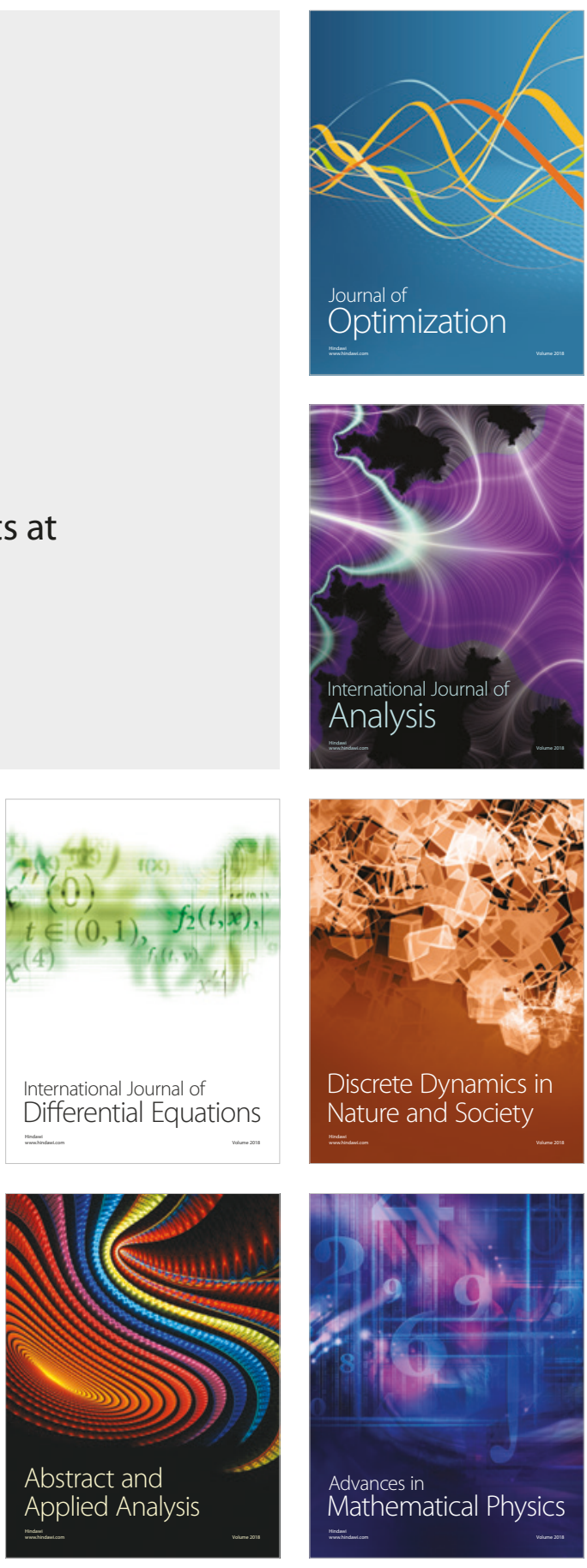